Pacific Journal of Mathematics

THE SECOND LIE ALGEBRA COHOMOLOGY GROUP AND

THY MODULES 


\title{
THE SECOND LIE ALGEBRA COHOMOLOGY GROUP AND WEYL MODULES
}

\author{
John Brendan Sullivan
}

Dedicated to Gerhard Hochschild on the occasion of his 65th birthday

The Lie algebra 1-cohomology of classical Lie algebras in characteristic $p$ is nonzero at some modules. In fact, Hochschild showed that the restricted 1-cohomology is nonzero. Here I will systematically produce Weyl modules at which the (unrestricted) 2-cohomology is nonzero. This will provide examples of nonsplit abelian extensions of Lie algebras by Weyl modules in characteristic $p$, where the Lie algebras are the reductions modulo $p$ of integral Chevalley forms of complex semisimple Lie algebras.

The method requires passages among cohomology groups for Lie algebras defined over the integers, rationals, and the prime field of characteristic $p$. In proving Theorem 1, we show that the 2 cohomology of an integral Lie algebra has nonzero $p$-torsion at a module whenever the reduced (modulo $p$ ) algebra has nonzero 1cohomology at the reduced module and the extension of the integral Lie algebra to the rationals has zero for its first and second cohomology at the extension of the module by the rationals. The tensor product of the integral cohomology group with the prime field is a nonzero piece of the cohomology group of the reduced Lie algebra. This will give the examples that we seek provided that we produce modules which satisfy the conditions given above for the groups of the reduced and rational algebras. The second condition is satisfied in the classical case since the rational Lie algebra will be semisimple. As for the first condition, in [4] I gave irreducible modules in characteristic $p$ at which the 1-cohomology is nonzero. I will show that the cohomology is also nonzero at a Weyl module associated to the irreducible module. The module that we seek for the integral Lie algebra is one that reduces modulo $p$ to the Weyl module. I have carried out this enterprise of showing that the cohomology is nonzero at the Weyl module only for the Lie algebra of the special linear group although I believe that it can be carried out generally.

In $\S 3, I$ take a particular one cocycle $g$ (constructed in [4]) and, working with an integral cochain which reduces to $g$, apply the coboundary operator to obtain explicitly a $p$-torsion element of the integral cohomology group.

I wish to thank Professor Hochschild for his kindness and generosity over the years. 
Notation. Let $L$ be a Lie algebra over the ring $Z$ of integers, let $V$ be a $Z$-module, and let $F$ be any commutative ring with unit. $V_{F}=V \otimes_{Z} F . \quad F_{p}$, the field with $p$ element $V_{p}=V_{F_{p}}$.

$L_{F}=L \otimes_{z} F$, an $F$-Lie algebra.

If $V$ is an $L$-module, then $V_{F}$ is an $L_{F}$-module.

$V_{F}^{L_{F}}=\left\{v \in V_{F} \mid k v=0\right.$ for all $\left.k \in L_{F}\right\}$, the space of invariants.

$C^{i}\left(L_{F}, V_{F}\right)=$ space of $i$-cochains for the Lie algebra cohomology of $L_{F}$ with values in $V_{F}$

$=\operatorname{Hom}_{F}\left({ }_{i} L_{F}, V_{F}\right)$, where ${ }_{\hat{i}} L_{F}$ is the $i$ th exterior power of $L_{F}$.

$Z^{i}\left(L_{F}, V_{F}\right)=$ space of $i$-cocycles.

$B^{i}\left(L_{F}, V_{F}\right)=$ space of $i$-coboundaries.

$H^{i}\left(L_{F}, V_{F}\right)=i$ th cohomology group.

$\delta^{i}=i$ th coboundary operator.

Let $\mathrm{sl}_{n+1, F}$ be the Lie algebra of $n+1$ by $n+1$ trace 0 matrices with entries in $F$. Let $E_{i j}$ be the trace zero matrix whose only nonzero entry is a 1 in the $i j$ th position. The $\left\{E_{i j}\right\}$ belong to the standard Chevalley basis for $\mathrm{sl}_{n+1, F}$.

$Y_{1}=E_{21}$.

$h_{F}=$ space of diagonal matrices in $\mathrm{sl}_{n+1, F}$.

$b_{F}=$ space of upper triangular matrices in $\mathrm{sl}_{n+1, F}$.

$b=$ space of upper triangular matrices in $\mathrm{sl}_{n+1, Z}$.

$\alpha_{i}$ is the element in the dual space of $h_{F}$ whose value at the matrix with diagonal entries $t_{j}$ is $t_{i}-t_{i+1}$.

1. Take a Lie algebra $L$ over the ring $Z$ of integers which is a free $Z$-module of finite rank. Let $V$ be any $L$-module which is a free $Z$-module of finite rank. Let $Q$ be the rational numbers.

Suppose that $L_{Q}$ is a semisimple Lie algebra and that the space of $L_{Q}$-invariants in $V_{Q}$ and the space of $L_{p}$-invariants in $V_{p}$ have the same dimension, say $m$. Then we have the following theorem.

TheOREM. If $H^{1}\left(L_{p}, V_{p}\right)$ is nonzero, then $H^{2}\left(L_{p}, V_{p}\right)$ is nonzero.

Proof. The proof is a comparison of the ranks and dimensions of various spaces coming from cochain complexes. The canonical map $C^{2}(L, V) \otimes_{z} F_{p} \rightarrow C^{2}\left(L_{p}, V_{p}\right)$ is a linear isomorphism since $L$ and $V$ are free $Z$-modules. Furthermore, the image of $Z^{2}(L, V) \otimes_{Z} F_{p}$ lies in $Z^{2}\left(L_{p}, V_{p}\right)$. Hence, the dimension of $Z^{2}\left(L_{p}, V_{p}\right)$ is at least as large as the rank of the free $Z$-module $Z^{2}(L, V)$, which equals the dimension of $Z^{2}\left(L_{Q}, V_{Q}\right)$.

The spaces $Z^{i}\left(L_{Q}, V_{Q}\right)$ and $B^{i}\left(L_{Q}, V_{Q}\right)$ coincide for $i=1,2$ since $H^{i}\left(L_{Q}, V_{Q}\right)=(0)$ for $i=1,2$ for a semisimple Lie algebra [2]. Therefore, $\operatorname{dim}\left(Z^{2}\left(L_{Q}, V_{Q}\right)\right)$ equals $\operatorname{dim}\left(C^{1}\left(L_{Q}, V_{Q}\right)\right)-\operatorname{dim}\left(V_{Q}\right)+m$. 
The dimension of $B^{2}\left(L_{p}, V_{p}\right)$, which equals $\operatorname{dim}\left(C^{1}\left(L_{p}, V_{p}\right)\right)$ $\operatorname{dim}\left(Z^{1}\left(L_{p}, V_{p}\right)\right)$, is less than $\operatorname{dim}\left(C^{1}\left(L_{p}, V_{p}\right)\right)-\operatorname{dim}\left(B^{1}\left(L_{p}, V_{p}\right)\right)$ since $H^{1}\left(L_{p}, V_{p}\right)$ is nonzero by hypothesis. Therefore, the dimension of $B^{2}\left(L_{p}, V_{p}\right)$ is less than $\operatorname{dim}\left(C^{1}\left(L_{p}, V_{p}\right)\right)-\operatorname{dim}\left(V_{p}\right)+m$, which equals $\operatorname{dim}\left(C^{1}\left(L_{Q}, V_{Q}\right)\right)-\operatorname{dim}\left(V_{Q}\right)+m$. By the conclusions of the previous two paragraphs, the dimension of $Z^{2}\left(L_{p}, V_{p}\right)$ exceeds the dimension of $B^{2}\left(L_{p}, V_{p}\right)$ and $H^{2}\left(L_{p}, V_{p}\right)$ is nonzero.

2. We will turn our attention specifically to the Lie algebra $\mathrm{Sl}_{n+1, F_{p}}$ of the special linear group $\mathrm{SL}_{n+1, F_{p}}$ over the prime field $F_{p}$. Let $\lambda_{i}$ be the character on the diagonal subgroup of $\mathrm{SL}_{n+1, F_{p}}$ whose value at the diagonal matrix with diagonal entries $\left\{t_{j}\right\}_{j}$ is $t_{1} \cdots t_{i}$. Let $W$ be the irreducible $\mathrm{SL}_{n+1, F_{p}}$-module whose highest weight is $(p-2) \lambda_{1}+\lambda_{2}$. $W$ will also stand for the space $W$ with the differential $\mathrm{sl}_{n+1, F_{p}}$-module structure. For any $\mathrm{SL}_{n+1, F_{p}}$-module $W^{\prime}$, we will give the weight of a line stable under the diagonal subgroup by a character on the diagonal subgroup, even though it may be the differential $\mathrm{sl}_{n+1, F_{p}}$-module structure of $W^{\prime}$ that is under consideration.

In [4], we showed that the one-cohomology of $\mathrm{sl}_{n+1, F_{p}}$ at $W$ is nonzero, except possibly for $\mathrm{sl}_{3, F_{3}}$. Below, we associate to $W$ a Weyl module $V_{p}$ and show that $\mathrm{sl}_{n+1, F_{p}}$ has nonzero two-cohomology at $V_{p}$, except possibly when $p=n+1$. Let $V_{Q}$ be the irreducible $\mathrm{SL}_{n+1, Q^{-}}$ module whose highest weight is $(p-2) \lambda_{1}+\lambda_{2}$. We construct a $Z$ form $V$ for $V_{Q}$ which is stable under the Kostant $Z$-form $U_{Z}$ for the universal enveloping algebra of $\mathrm{sl}_{n+1, Q}$ (relative to the standard basis for $\mathrm{sl}_{n+1, Q}$ ) and whose reduction modulo $p, V_{p}$, contains $W$ as a submodule for its $\mathrm{SL}_{n+1, F_{p}}$-module structure.

The standard $Z$-form of $V_{Q}$ is the $U_{Z}$-submodule that is generated from a highest weight vector $[1, A]$. The $Z$-form $V$ is the variant that is produced as follows. Take a lowest weight vector $v^{*}$ from the $\mathrm{SL}_{n+1, Q}$-module $V_{Q}^{*}$ that is contragradient to $V_{Q}$. The weight of $v^{*}$, reduced modulo $p$, equals the lowest weight of $W^{*}$. The $U_{Z}$-module $U_{Z} v^{*}$ is a $Z$-form for $V_{Q}^{*}$ for which the $\mathrm{SL}_{n+1, F}$-module $U_{Z} v^{*} \otimes_{Z} F_{p}$ contains a unique maximal proper submodule. The quotient module is isomorphic to $W^{*}$ since the lowest weight of the quotient module is the weight of $v^{*}$, reduced modulo $p$. The $U_{Z^{-}}$ module $V=\operatorname{Hom}\left(U_{Z} v^{*}, Z\right)$ is a $Z$-form for $V_{Q}$ and the $\mathrm{SL}_{n+1, F}$-module $V_{p}=V \otimes_{z} F_{p}$, which is contragradient to $U_{Z} v^{*} \otimes_{z} F_{p}$, has a unique irreducible submodule. This submodule is isomorphic to $W$.

The following lemma and its use in proving the proposition below were suggested by John Ballard. The proof has been consigned to the last section.

Lemma. If $p \neq n+1$, then $V_{p}$ contains no weight $p \lambda$ where $\lambda$ 
is dominant.

Proposition. If $p \neq n+1$, then $H^{1}\left(\mathrm{sl}_{n+1, F}, V_{p}\right)$ is nonzero.

Proof. From the long exact sequence of cohomology associated with the exact sequence $0 \rightarrow W \rightarrow V_{p} \rightarrow V_{p} / W \rightarrow 0$, take the exact sequence $\left(V_{p} / W\right)^{s 1_{n+1}, F_{p}} \rightarrow H^{1}\left(\mathrm{sl}_{n+1, F_{p}}, W\right) \rightarrow H^{1}\left(\mathrm{sl}_{n+1, F_{p}}, V_{p}\right)$. It will be enough to show that the first term is (0) since $H^{1}\left(\mathrm{sl}_{n+1, F_{p}}, W\right)$ is nonzero. $\left(V_{p} / W\right)^{\mathrm{s} 1_{n+1}, F_{p}}$ is an $\mathrm{SL}_{n+1, F_{p}}$-submodule of $V_{p} / W$, which is trivial as an $\mathrm{sl}_{n+1, F}$-module. A highest weight vector of this submodule would be dominant weight of the form $p \lambda$, which the lemma excludes from $V_{p}$. Therefore, $V_{p} / W$ has (0) as its space of invariants.

THEOREM. If $p \neq n+1$, then $H^{2}\left(\mathrm{sl}_{n+1, F_{p},-}\right)$ is nonzero at the Weyl module $V_{p}$.

Proof. In the theorem of section one, the hypothesis that $H^{1}\left(\mathrm{sl}_{n+1, F_{p}}, V_{p}\right)$ be nonzero is satisfied by the proposition above. The hypothesis that the spaces of invariants of $V_{Q}$ and $V_{p}$ have the same dimension is satisfied. In fact, $V_{p}^{\mathrm{sl}_{n+1}, F_{p}}$ is zero, by the argument used in proving the proposition. Therefore, $V_{Q}^{\text {s1 }} n+1, Q$ is zero as well. We may conclude that $H^{2}\left(\mathrm{sl}_{n+1, F_{p}}, V_{p}\right)$ is nonzero.

3. We will show the origin of one nonzero cohomology class in $H^{2}\left(\mathrm{sl}_{n+1, F_{p}}, V_{p}\right)$.

Denote $\mathrm{sl}_{n+1, Z}$ by $L$, denote the weight $(p-2) \lambda_{1}+\lambda_{2}$ by $\beta$, and let $W$ and $V$ be the modules given in $\S 2$. In [4], we constructed a noncobounding one cocycle $g$ for $L_{p}$ with values in $W$ with the properties that $g$ is zero on $b_{p}$ and $g\left(Y_{1}\right)$ is a highest weight vector in $W$. The reduction modulo $p$ of the unique line of weight $\beta$ in $V$ in the unique line of weight $\beta$ in $V_{p}$. Choose any cochain $g_{z}$ in $C^{1}(L, V)$ for which $g_{Z} \otimes_{Z} F_{p}=g, g_{z}(b)=(0)$, and $g_{Z}\left(Y_{1}\right)$ is a vector of weight $\beta$. We will show that the 2-cochain $f=\left((1 / p) \delta^{1}\left(g_{z}\right)\right) \bigotimes_{z} F_{p}$ represents a nonzero cohomology class for $L_{p}$ with values in $V_{p}$. Since $\delta^{1} g=0, \delta^{1} g_{z}$ is divisible by $p$ in $C^{2}(L, V)$ and we may form the cochain $(1 / p)\left(\delta^{1} g_{z}\right)$ in $C^{2}(L, V)$. This is a cocycle since $\delta^{2}\left((1 / p)\left(\delta^{1}\left(g_{z}\right)\right)\right)=(1 / p)\left(\delta^{2} \delta^{1} g_{z}\right)=0$ and it maps $b \wedge b$ to 0 since $g_{z}(b)=$ (0). Therefore, $f=\left((1 / p)\left(\delta^{1} g_{z}\right)\right) \otimes_{z} F_{p}$ is a two cocycle for $L_{p}$ with values in $V_{p}$, which maps $b_{p} \wedge b_{p}$ to 0 . We show that $f$ is not a coboundary.

Suppose that $f$ were the coboundary $\delta^{1} u$ for some cochain $u: L_{p} \rightarrow V_{p}$. The restriction of $u$ to $b_{p}$ is a cocycle since $f\left(b_{p} \wedge b_{p}\right)=(0)$. By the lemma of $\S 3.1$ of [4], the canonical image of $H_{b}^{1}\left(b_{p}, V_{p}\right)$ in $H^{1}\left(h_{p}, V_{p}\right)$ is contained in the image of $H^{1}\left(h_{p}, V_{p}^{b} p\right)$ in $H^{1}\left(h_{p}, V_{p}\right)$; 
here, since $V_{p}^{b}=(0),(0)$ is the image of $H^{1}\left(b_{p}, V_{p}\right)$ in $H^{1}\left(h_{p}, V_{p}\right)$. Therefore, there is an element $v$ of $V_{p}$ such that $u-\delta^{0} v$ is zero on $h_{p}$. Now use the symbol $u$ for the 'normalized' cochain $u-\delta^{0} v$. We show that $f$ cannot equal $\delta^{1} u$ by showing that $f\left(Y_{1} \wedge H_{1}\right)$ has a nonzero component of weight $\beta$, while $\delta^{1} u\left(Y_{1} \wedge H_{1}\right)$ has zero for its component of weight $\beta$. $\delta^{1} u\left(\mathrm{Y}_{1} \wedge H_{1}\right)=\left(-2-H_{1}\right) u\left(\mathrm{Y}_{1}\right)$ has zero as its component of weight $\beta$ since $-2-H_{1}$ annihilates vectors of weight $\beta$. By the construction of $g_{Z}, g_{Z}\left(Y_{1}\right)$ has as its component of weight $\beta$ a vector $x$ which is nonzero modulo $p$. Write $g_{z}\left(Y_{1}\right)=$ $x+y$. Then $(1 / p) \hat{o}^{1} g_{Z}\left(Y_{1} \wedge H_{1}\right)=(1 / p)\left(-2-H_{1}\right) g_{Z}\left(Y_{1}\right)=x+(1 / p)\left(-2-H_{1}\right) y$, reduced modulo $p$, has a nonzero component of weight $\beta$.

Thus, we see that $(1 / p) \delta^{1} g_{Z}$ represents a cohomology class in $H^{2}\left(\mathrm{sl}_{n+1}, z, V\right)$ whose reduction modulo $p$ gives a nonzero element of $H^{2}\left(\mathrm{sl}_{n+1, F}, V_{p}\right)$. We wish to remark that the cohomology class of $f$ does not depend on the choice of lifting of $g$ to an integral cochain. In fact, if $g_{z}^{\prime}$ is another cochain such that $g_{Z}^{\prime} \boldsymbol{\otimes}_{Z} F_{p}=g$, then $(1 / p) \delta^{1} g_{Z}-(1 / p) \delta^{1} g_{Z}^{\prime}$ is the two coboundary $\delta^{1}\left((1 / p)\left(g_{Z}-g_{Z}^{\prime}\right)\right)$, and $(1 / p) \delta^{1} g_{Z}$ and $(1 / p) \delta^{1} g_{Z}^{\prime}$ represent the same class in $H^{2}\left(\mathrm{sl}_{n+1, Z}, V\right)$.

It would be good to know if $H^{2}\left(\mathrm{sl}_{n+1, F_{p}}, W\right)$ is also nonzero. In particular, does the cohomology class of the element $f$ lie in the canonical image of $H^{2}\left(\mathrm{sl}_{n+1, k}, W\right)$ in $H^{2}\left(\mathrm{sl}_{n+1, F_{p}}, V_{p}\right)$ ?

4. Here we give a proof of the lemma of $\S 2$. The weights of the $\mathrm{SL}_{n+1, Q}$-module $V_{Q}$ have the form $\beta-\sum a_{i} \alpha_{i}$, where $\beta=$ $(p-2) \lambda_{1}+\lambda_{2}$ and the $a_{i}$ are integers in the range $0 \leqq a_{1}, a_{n} \leqq p-1$, and $0 \leqq a_{i} \leqq p$ for $i \neq 1$, n, c.f. $[4,3.3]$. The weights of the $\mathrm{SL}_{n+1, i^{\prime}}$-module $V_{p}$ inherit the same property. The roots $\alpha_{i}$ are expressed in terms of the fundamental dominant weights $\lambda_{2}$ as follows: $\alpha_{1}=2 \lambda_{1}-\lambda_{2}, \alpha_{n}=2 \lambda_{n}-\lambda_{n-1}$, and $\alpha_{i}=2 \lambda_{i}-\lambda_{i-1}-\lambda_{\imath+1}$ for $i \neq 1, n$.

Suppose that $p \lambda=\beta-\sum a_{\imath} \alpha_{i}$ is a weight of $V_{p}$, where $\lambda$ is a dominant weight $\sum b_{i} \lambda_{i}, b_{i} \geqq 0$. Let $j$ be the highest index such that $a_{j}$ is nonzero. We will show that $j$ must be $n$ and that $p=$ $n+1$. If $n>j>2$, then the coefficient of $\lambda_{j+1}$ in $\beta-\sum a_{i} \alpha_{i}=p \lambda$ is $p b_{j+1}=a_{j}$, and $a_{j}=p$ since $0<a_{j} \leqq p$. The coefficient of $\lambda_{j}$ is $p b_{j}=-2 a_{j}+a_{j-1}=-2 p+a_{j-1}$. This is not possible since $b_{j}$ is nonnegative and $a_{j-1} \leqq p$. A similar argument shows that it is not possible to have $n>j$ when $j=1$ or 2 . Hence, $j$ must be $n$.

The coefficient of $\lambda_{n}$ in $p \lambda=\beta-\sum a_{i} \alpha_{2}$ is $p b_{n}=-2 a_{n}+a_{n-1}$. Since $b_{n}$ is nonnegative, $a_{n}$ is positive, and $a_{n-1} \leqq p, a_{n-1}$ must equal $2 a_{n}$. More generally, we will check that the formula $a_{i}=(n+1-i) a_{n}$ holds for any $i \geqq 2$. Suppose that it holds for all $i \geqq k$, where $k$ is an index in the range $n-1>k>2$. The coefficient of $\lambda_{k}$ is $p b_{k}=a_{k-1}+a_{k+1}-2 a_{k}=a_{k-1}-(n+2-k) a_{n}$. As above for $k=n-1$, 
we conclude that $a_{k-1}=(n+2-k) a_{n}$. By the formula, the coefficient of $\lambda_{2}$ is $p b_{2}=1+a_{1}+a_{3}-2 a_{2}=1+a_{1}-n a_{n}$ and so, $a_{1}=n a_{n}-1$. The coefficient of $\lambda_{1}$ is $p b_{1}=p-2+a_{2}-2 a_{1}=p-(n+1) a_{n}$, and so, $p=(n+1) a_{n}$. We may conclude that $p=n+1, a_{n}=1, \beta=$ $(n-1) \alpha_{1}+\sum_{i=2}^{n}(n+1-i) \alpha_{i}$, and that only in characteristic $p=$ $n+1$ can $V_{p}$ have a dominant weight of the form $p \lambda$.

\section{REFERENCES}

1. A. Borel et al., Seminar on Algebraic Groups and Related Finite Groups, Lecture Notes in Mathematics, Springer-Verlag, Heidelberg, 1970, Vol. 131.

2. C. Chevalley and S. Eilenberg, Cohomology theory of Lie groups and Lie algebras, TAMS, 63 (1948), 85-124.

3. G. Hochschild, Representations of restricted Lie algebras of characteristic p, Proc. Amer. Math. Soc., 5 (1954), 603-605.

4. J. Sullivan, Lie algebra cohomology at irreducible modules, Illinois J. Math., 23 (1979), 363-373.

Received June 19, 178. Supported in part by a National Science Foundation Grant.

UNIVERSITY OF WASHINGTON

SeAtTLE, WA 98195 


\section{PACIFIC JOURNAL OF MATHEMATICS}

\section{EDITORS}

DONALD BABBITT (Managing Editor)

University of Galifornia

Los Angeles, California 90024

HUGo RossI

University of Utah

Salt Lake City, UT 84112

C. C. MOORE AND ANDREW OGG

University of California

Berkeley, CA 94720
J. DUGUNDJI

Department of Mathematics University of Southern California Los Angeles, California 90007

R. FINN AND J. MILGRAM Stanford University Stanford, California 94305

\section{ASSOCIATE EDITORS}

E. F. BECKENBACH

B. H. NEUMANN

F. WOLF

K. YosHIDA

\section{SUPPORTING INSTITUTIONS}

UNIVERSITY OF BRITISH COLUMBIA CALIFORNIA INSTITUTE OF TECHNOLOGY UNIVERSITY OF CALIFORNIA MONTANA STATE UNIVERSITY UNIVERSITY OF NEVADA, RENO NEW MEXICO STATE UNIVERSITY OREGON STATE UNIVERSITY UNIVERSITY OF OREGON
UNIVERSITY OF SOUTHERN CALIFONIA STANFORD UNIVERSITY UNIVERSITY OF HAWAII UNIVERSITY OF TOKYO UNIVERSITY OF UTAH WASHINGTON STATE UNIVERSITY UNIVERSITY OF WASHINGTON 


\section{Pacific Journal of Mathematics}

\section{Vol. 86, No. $1 \quad$ November, 1980}

Gert Einar Torsten Almkvist, Invariants, mostly old ones .............. 1

Hyman Bass, Groups of integral representation type ................ 15

A. Białynicki-Birula, On action of SL(2) on complete algebraic

varieties........................................

Frederick Paul Greenleaf and Martin Allen Moskowitz, Groups of

automorphisms of Lie groups: density properties, bounded orbits, and

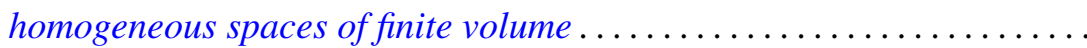

Raymond Taylor Hoobler, A cohomological interpretation of Brauer groups

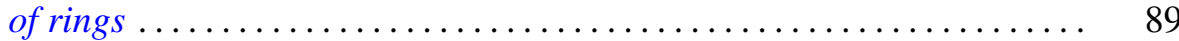

Irving Kaplansky, Superalgebras ........................ 93

Jerrold Lewis Kleinstein and Alex I. Rosenberg, Succinct and

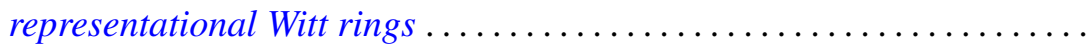

E. R. Kolchin, On universal extensions of differential fields ............ 139

Andy R. Magid, Analytic subgroups of affine algebraic groups. II ....... 145

Calvin Cooper Moore, The Mautner phenomenon for general unitary

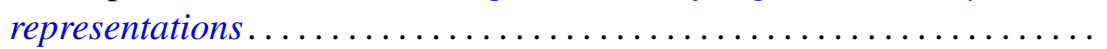

George Daniel Mostow, On a remarkable class of polyhedra in complex hyperbolic space ................................ 171

Brian Lee Peterson, Extensions of pro-affine algebraic groups. II . . . . . . 277

John Henry Reinoehl, Lie algebras and affine algebraic groups......... 287

Maxwell Alexander Rosenlicht, Differential valuations .

John Brendan Sullivan, The second Lie algebra cohomology group and Weyl modules..................................... 321

Moss Eisenberg Sweedler, Right derivations and right differential

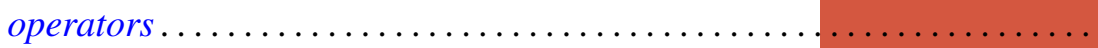

Bostwick Frampton Wyman, Time varying linear discrete-time systems. II. Duality.................................. 